\title{
Le potassium administré comme supplément et biostimulateur dans l'alimentation de la volaille I. Influence du potassium sur le développement des poulets de chair
}

\author{
Gh. SALAJAN, O. POPA, Ludmila MOSOLOVA, V. DANKANITS \\ Institutul Agronomic, Facultatea de Zootehnie \\ Str. Manastur nr. 3, 3400 Cluj-Napoca (Roumanie)
}

\begin{abstract}
Résumé
L'influence du potassium administré comme supplément dans l'alimentation des poulets de chair a été étudié : on en a conclu que, par une hausse de 0,10 p. 100 du taux de potassium dans le régime $(+100 \mathrm{~g} \mathrm{~K} / 100 \mathrm{~kg}$ de l'aliment composé) en comparaison avec le niveau actuellement utilisé dans les élevages avicoles intensifs, on fait augmenter de 3,96-4,12 p. 100 le poids corporel des poulets sur l'ensemble du cycie de croissance, et de 3,02-6,76 p. 100 le degré de transformation des aliments.

Les meilleurs résultats ont été obtenus pendant les 30 premiers jours d'âge. Par rapport au taux actuel de 0,63 et 0,78 p. 100 , on a déduit que le niveau optimum du potassium dans la ration pour les poulets de chair est de 0,72 p. 100 pendant la période d'âge allant de 1 à 30 jours et de 0,88 p. 100 ensuite jusqu'à l'abattage.
\end{abstract}

\section{Introduction}

Certains physiologistes, parmi lesquels on peut citer Cristea et al. (1978), KolB \& Gurtler (1971), Kolb (1974), ont montré que le potassium est indispensable pour l'organisme, de par les rôles qu'il joue dans le métabolisme et dans les activités vitales de la cellule. Baia (1972), Martin et Mihar (1979) estiment que le taux nécessaire de $\mathrm{K}$ pour les animaux est de 0,2-0,4 p. 100 dans la ration.

Les recherches concernant l'incidence du potassium sur la production des animaux domestiques ont été négligées à cause du taux assez réduit de $\mathrm{K}$ nécessaire et également du fait de l'existence dans les aliments courants d'un excédent de cet élément.

A propos de ce problème, Gürtler et al. (1975), KolB et al. (1971), KolB (1974) ont constaté que les carcasses de volaille contiennent un taux plus élevé de potassium en comparaison avec les autres animaux, ce qui pourrait signifier des besoins plus grands. A l'appui de cette idée viennent les résultats obtenus par SuppleE (1965) et 
WiEsner (1970) cités par Martin et al. (1980), qui ont montré l'augmentation du poids corporel des poulets de chair et des dindonneaux alors que le taux de potassium était augmenté jusqu’à 0,60 p. 100 dans la ration.

TABLEAU 1

Composition centésimale des aliments expérimentaux pour les poulets de chair Composition of experimental broiler diets

\begin{tabular}{|c|c|c|}
\hline \multirow{2}{*}{$\begin{array}{c}\text { Matières premières ou composants } \\
\text { (Ingredients) }\end{array}$} & \multicolumn{2}{|c|}{$\begin{array}{l}\text { Période expérimentale } \\
\text { Age of broilers }\end{array}$} \\
\hline & $\begin{array}{c}\mathrm{I}^{\mathrm{re}} \\
(1-30 \text { jours }) \\
\text { (days) }\end{array}$ & $\begin{array}{c}\mathrm{II}^{\mathrm{e}} \\
\text { (31-56 jours) } \\
\text { (days) }\end{array}$ \\
\hline \multicolumn{3}{|l|}{ Composition (p. 100 du poids) (\% of weight) } \\
\hline Maïs $($ Maize $) \ldots \ldots \ldots \ldots \ldots \ldots \ldots \ldots$ & 55,30 & 55,70 \\
\hline Blé fourrager (Fodder wheat) $\ldots \ldots \ldots \ldots$ & 一 & 10,00 \\
\hline Tourteau de tournesol (Sunflower oil-meal) & - & 5,00 \\
\hline Soja grossièrement moulu (Soybean meal) .. & 28,40 & 19,50 \\
\hline Farine de viande (Meat meal) $\ldots \ldots \ldots \ldots$ & - & 2,00 \\
\hline Farine de poisson (Fish meal) $\ldots \ldots \ldots \ldots$ & 5,50 & 3,00 \\
\hline Poudre de lait écrémé (Dried skimmilk) ...... & 4,00 & 一 \\
\hline Prémélange de méthionine (Methionine premix) & 0,80 & 0,40 \\
\hline Prémélange de choline (Choline premix) .... & 2,00 & 1,20 \\
\hline Concentré de lysine (Lysine concentrate) .... & 0,30 & - \\
\hline Craie broyée (Feed-grade limestone) $\ldots \ldots \ldots$ & 1,50 & 1,00 \\
\hline Phosphate bicalcique (Dicalcium phosphate). & 1,00 & 1,00 \\
\hline Chlorure de sodium (Sodium chloride) ........ & 0,20 & 0,20 \\
\hline Zoofort $A_{1} \ldots \ldots \ldots \ldots \ldots \ldots \ldots \ldots \ldots$ & 1,00 & - \\
\hline 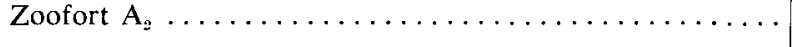 & 一 & 1,00 \\
\hline Total $\ldots \ldots \ldots \ldots \ldots \ldots \ldots \ldots \ldots \ldots \ldots \ldots \ldots \ldots \ldots \ldots \ldots \ldots$ & 100,00 & 100,00 \\
\hline \multicolumn{3}{|l|}{$\begin{array}{c}\text { Caractéristiques nutritives } \\
\text { Nutritive characteristics }\end{array}$} \\
\hline Energie nette $($ Net energy) $(\mathrm{Kcal} / \mathrm{kg}) \ldots \ldots \ldots \ldots \ldots$ & 1950 & 2126 \\
\hline Matières azotées totales (Crude protein) $(\%)$. & 21,60 & 20,00 \\
\hline Lysine $(\%) \ldots \ldots \ldots \ldots \ldots \ldots \ldots \ldots \ldots \ldots$ & 1,12 & 0,94 \\
\hline Méthionine $(\%) \ldots \ldots \ldots \ldots \ldots \ldots \ldots \ldots$ & 0,45 & 0,41 \\
\hline Cystine $(\%) \quad \ldots \ldots \ldots \ldots \ldots \ldots \ldots \ldots$ & 0,33 & 0,26 \\
\hline Tryptophane $(\%) \quad \ldots \ldots \ldots$ & 0,20 & 0,18 \\
\hline Calcium $(\%), \ldots \ldots \ldots \ldots \ldots \ldots \ldots \ldots \ldots$ & 110 & 1,00 \\
\hline 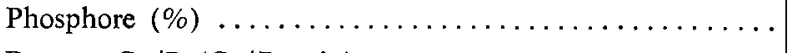 & 1,00 & 0,85 \\
\hline 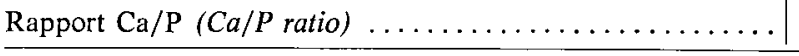 & 1,10 & 1,18 \\
\hline
\end{tabular}


Ces considérations nous ont conduit à entreprendre des recherches plus approfondies sur l'influence du potassium administré comme complément de la ration sur la croissance des poulets de chair.

\section{Matériel et méthodes}

Les recherches ont été effectuées sur des poulets hybrides Robro, répartis en lots de 50 poulets chacun, en deux répétitions $\left(R_{1}\right.$ et $\left.R_{22}\right)$ de 4 lots chacune, élevés dans le même poulailler.

Les poulets de la $1^{\text {re }}$ répétition (lots 1 à 4 ) ont été élevés en batterie, tandis que ceux de la $2^{\mathrm{e}}$ répétition (lots 5 à 8 ) sur plancher. Toutes les conditions de microclimat, ainsi que les soins et la technique d'élevage ont été identiques pour tous les poulets des deux répétitions.

La durée expérimentale a été de 56 jours, divisée en deux périodes d'âge : la $1^{\text {re }}$ de 1 jour à 30 jours et la $2^{\circ}$ de 31 à 56 jours. Tous les poulets ont été alimentés à volonté avec le même aliment composé (tableau 1).

\section{TABLEaU 2}

Teneur en potassium du régime expérimental des poulets de chair Potassium content of experimental diets for broilers

\begin{tabular}{|c|c|}
\hline Matières premières (Ingredients) & $\begin{array}{c}\text { Teneur en } \mathrm{K} \\
\text { (p. } 100 \text { du poids) } \\
K \text { content } \\
(\% \text { of weight })\end{array}$ \\
\hline Maïs (Maize) & 0,31 \\
\hline Blé (Wheat) $\ldots \ldots \ldots \ldots \ldots \ldots \ldots$ & 0,40 \\
\hline Tourteau de tournesol (Sunflower meal). & 1,20 \\
\hline Soja grossièrement moulu (Soybean meal) & 2,00 \\
\hline Farine de viande (Meat meal) $\ldots . . . \ldots$ & 1,40 \\
\hline Farine de poisson (Fish meal) & 1,36 \\
\hline Poudre de lait écrémé (Dried skimmilk) ....... & 1,16 \\
\hline 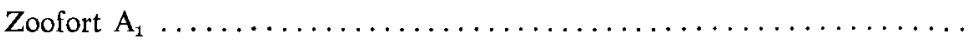 & 1,74 \\
\hline 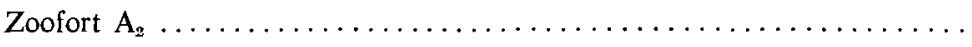 & 1,60 \\
\hline Régime combiné 21-1 (Compound diet 21-1), $1^{\text {re }}$ période (1st period) .. & 0,62 \\
\hline Régime combiné $21-2$ (Compound diet $21-2), 2^{\circ}$ période (2nd period) & 0,78 \\
\hline
\end{tabular}

Tout au début, on a déterminé la teneur en potassium des matières premières incorporées dans l'aliment composé, examinées séparément et puis celle de l'aliment composé dans son ensemble (tableau 2) en utilisant la méthode de la spectrophotométrie de flamme. 


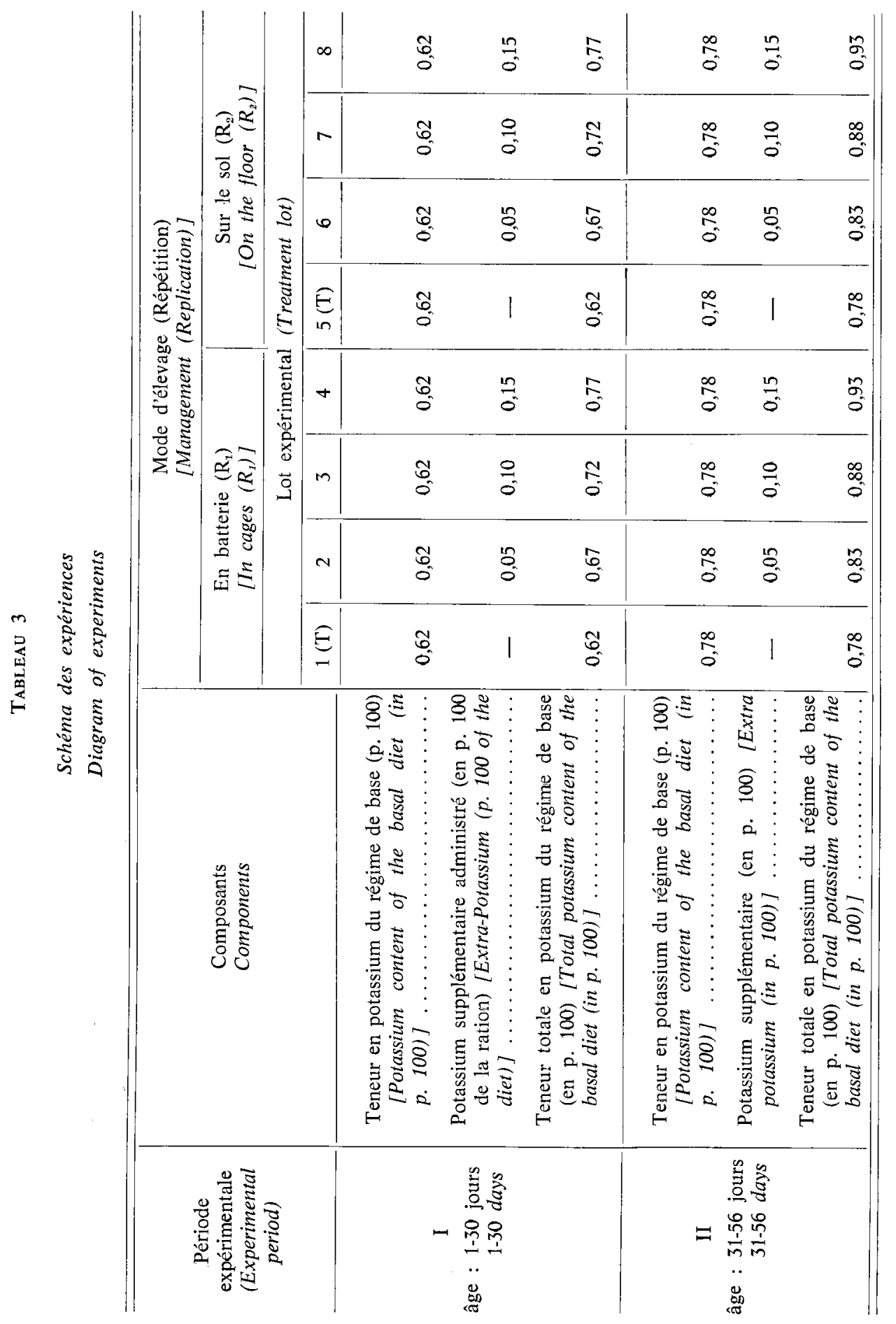


Après avoir déterminé la teneur en $\mathrm{K}$ de l'aliment composé de base $(=0,62$ p. 100 dans la $1^{\text {re }}$ période et 0,78 p. 100 dans la $2^{\mathrm{e}}$ ), on a étudié les compléments en cet élément pour chaque lot (tableau 3).

Les poulets des lots témoins ( 1 pour la $1^{\text {re }}$ répétition et 5 pour la $2^{\mathrm{e}}$ ) ont été alimentés avec l'aliment composé de base seul (sans additifs), tandis que pour les poulets des autres lots on a ajouté du potassium, soit : 0,05 p. 100 pour les lots 2 et $6,0,10$ p. 100 pour les lots 3 et 7 , et 0,15 p. 100 pour les lots 4 et 8 .

La source de potassium utilisée était le chlorure de potassium $(\mathrm{kcl})$ à 52,44 p. 100 de matière sèche. Périodiquement, on a enregistré l'évolution du poids corporel, le gain moyen journalier et la consommation spécifique d'aliments.

Les données expérimentales ont été interprétées statistiquement par la méthode de l'analyse de variance.

\section{Résultats et discussion}

Initialement, le poids corporel moyen des poulets a été pratiquement identique pour tous les lots (tableau 4), les différences entre ceux-ci n'étant pas significatives $(\mathrm{p}>0,05)$.

Au cours des expériences, les poulets recevant un supplément de potassium, c'est-à-dire les lots $2,3,4$ et respectivement 6,7 et 8 , ont eu des gains de poids corporel plus grands, comparativement aux lots témoins 1 et 5 , quoique les différences n'aient pas été significatives ( $\mathrm{p}<0,05$ ou 0,01 ) sauf pour les lots 3 et 4 (comparés avec le lot $1 \mathrm{~T}$ ) et pour les lots 7 et 8 (comparés avec le lot $5 \mathrm{~T}$ ), et seulement pendant les 30 premiers jours d'âge.

Les résultats moyens obtenus, aussi bien sur les périodes d’âge considérées séparément que sur l'ensemble du cycle expérimental (tableau 5), ont mis en évidence l'efficacité du potassium administré comme complément dans l'alimentation des poulets des lots 3 et 4,7 et 8 .

Dans la pratique, les différences entre les variantes des lots 3 et 7 (avec un supplément de $100 \mathrm{~g} \mathrm{~K} / 100 \mathrm{~kg}$ aliments), ainsi que des lots 4 et 8 (avec un supplément de $150 \mathrm{~g} \mathrm{~K} / 100 \mathrm{~kg}$ aliments) sont faibles, n'étant pas statistiquement significatives $(\mathrm{p}>0,05)$ de sorte que la variante la plus avantageuse reste celle des lots 3 et 7 , chez lesquels le complément en potassium a été assez faible $(100 \mathrm{~g} / 100 \mathrm{~kg}$ d'aliments).

Dans le cas de cette dernière variante, le gain moyen journalier a augmenté de 3,35 à 6,62 p. $100(\mathrm{p}<0,05)$ dans la $1^{\text {re }}$ période d'âge et de 2,57 à 2,96 p. 100 dans la $2^{\text {e }}$ période, parallèlement à une réduction de la consommation spécifique d'aliments de 3,88 à 10,96 p. 100 et de 2,08 à 5,17 p. 100 , respectivement.

Sur l'ensemble du cycle expérimental, les lots 3 et 7 ont obtenu une augmentation de gain moyen journalier de 3,96-4,12 p. 100 et une réduction moyenne de la consommation d'aliments par $\mathrm{kg}$ de gain de 3,03-6,76 p. 100 .

Les résultats enregistrés dans le cadre de deux répétitions ont été semblables, ce qui prouve l'efficacité du potassium administré comme complément à la ration, dans l'un comme dans l'autre mode d'élevage des poulets de chair (en batterie ou sur sol). 


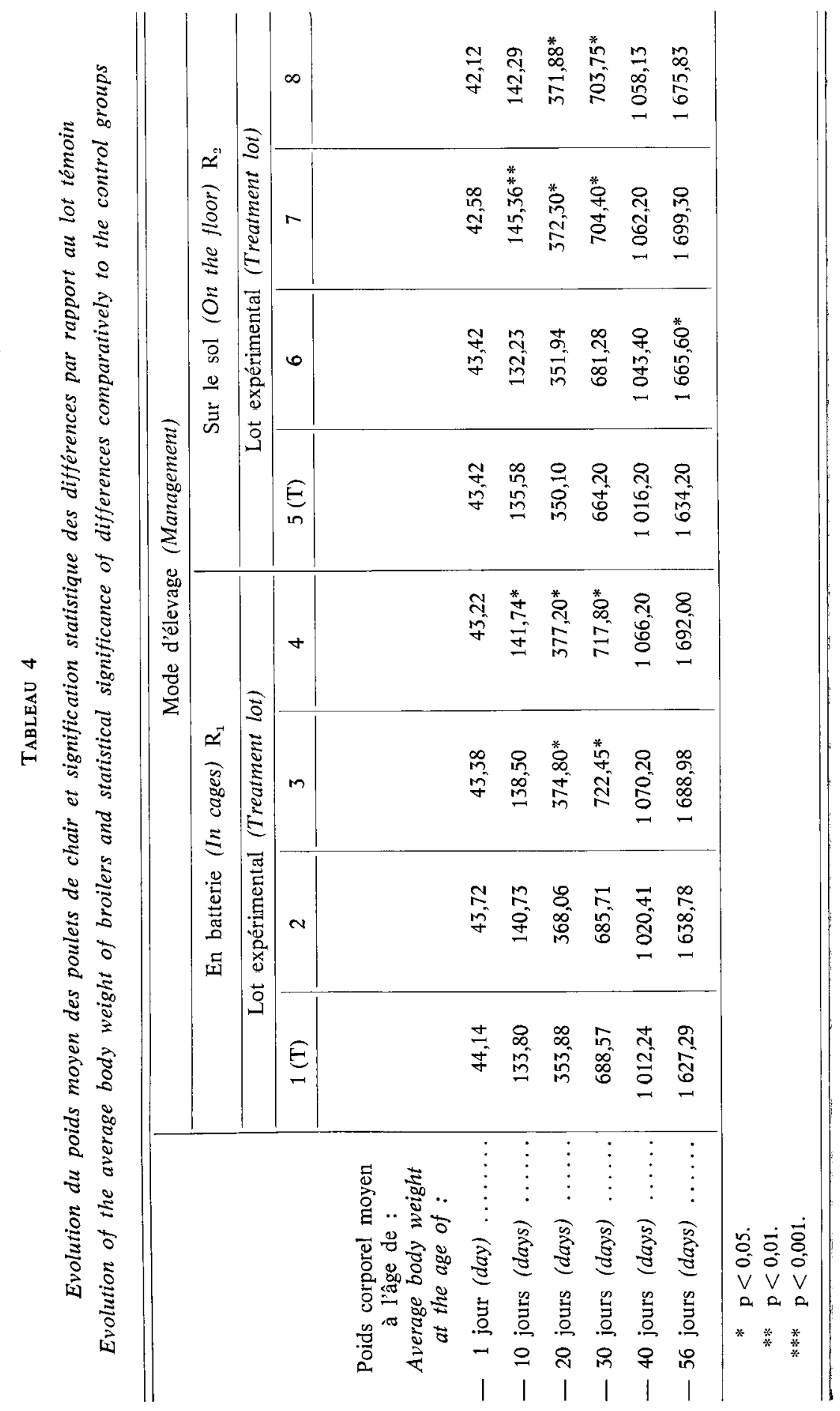




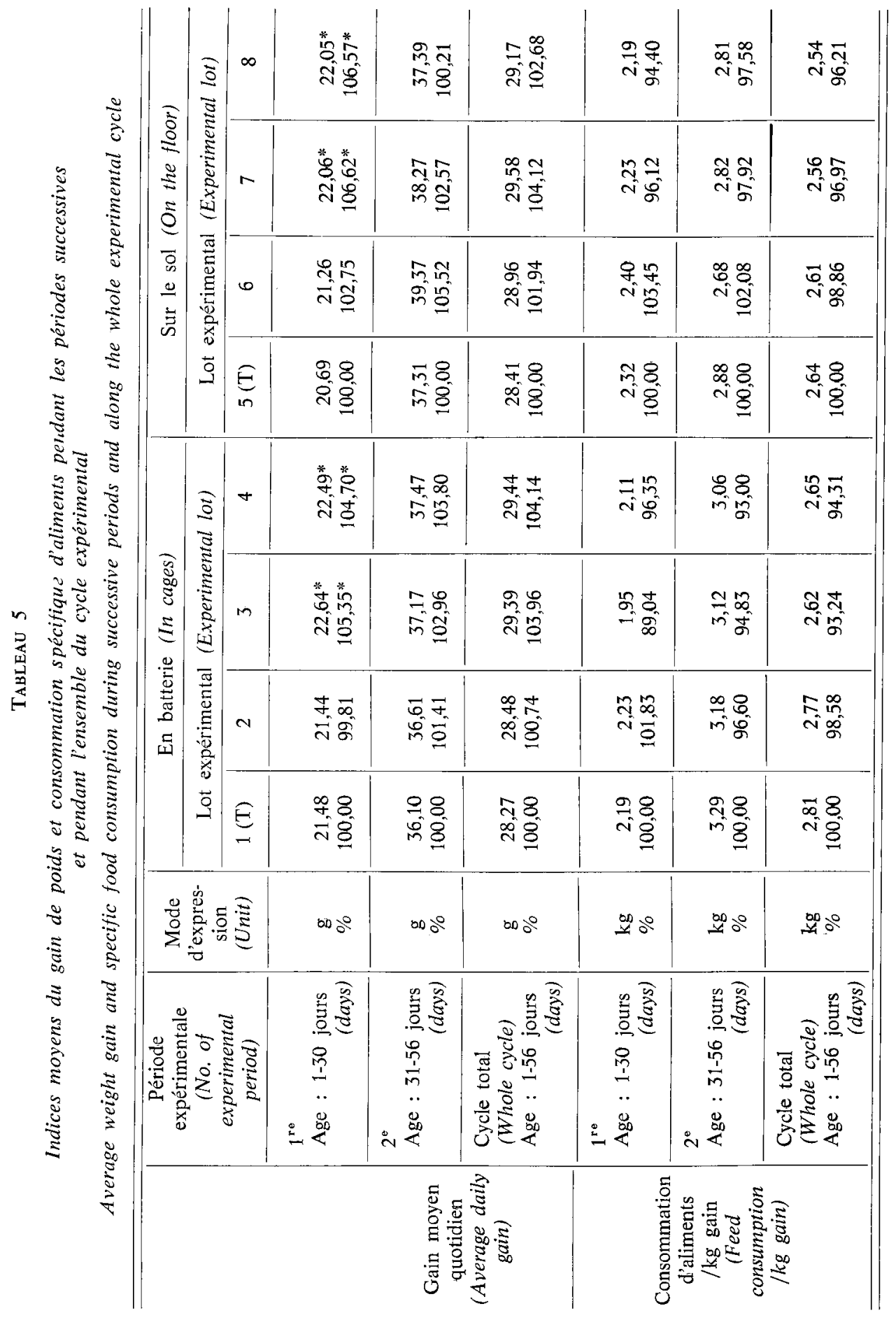


Sur la base de ces données, on peut estimer que les besoins en $\mathbf{K}$ des poulets de chair ne peuvent pas être satisfaits par le régime alimentaire habituel ; il faudrait donc y ajouter des sources spéciales de potassium jusqu'à un taux de 0,72 p. 100 de 1 à 30 jours d'âge et de 0,88 p. 100 par la suite.

Dans la pratique de l'élevage on peut supplémenter le régime d'aliments composés généralement utilisé, par une addition de 0,1 p. 100 de chlorure de potassium pendant toute la durée de la croissance des poulets, et tout spécialement les 30 premiers jours.

\title{
Conclusions
}

1. Le niveau du potassium des aliments composés usuels ne satisfait pas les besoins des poulets de chair; il est donc nécessaire d'y remédier par un supplément de cet élément.

2. L'administration du $\mathrm{K}$ comme complément aux aliments composés stimule la croissance des poulets de chair et augmente le degré de conversion des aliments.

3. On peut penser que le taux supplémentaire optimal de $\mathrm{K}$ dans l'alimentation des poulets de chair est de 0,1 p. 100 , ce qui permettrait d'obtenir une augmentation du gain moyen journalier corporel de 3,96-4,12 p. 100 et une réduction de la consommation d'aliments de 3,03-6,76 p. 100 par $\mathrm{kg}$ de gain.

4. Sur la base de ces résultats, on peut penser que le niveau optimal de potassium pour les poulets de chair est, en général, de 0,72 p. 100 dans la période d'âge allant de 1 à 30 jours et de 0,88 p. 100 ensuite, jusqu'à l'abattage.

Accepté pour publication en décembre 1980.

\begin{abstract}
Summary
Potassium supplementation as a biostimulator of poultry feeding

I. - Effect of extra-potassium on the growth rate of broilers
\end{abstract}

Experiments were conducted in order to study the effect of potassium supplementation on the growth rate of broilers. After having investigated several variances it was concluded that the increase in the dietary level of potassium by $0.1 \mathrm{p} .100(=100 \mathrm{~g} \mathrm{~K} / 100 \mathrm{~kg}$ feed $)$ comparatively to the usual standard level, gave the best results by increasing the body weight of broilers from 3.96 p. 100 to 4.12 p. 100 and improving the feed conversion ratio from 3.02 p. 100 to 6.76 p. 100 . The results were much better in the first 30 days of age.

Thus, the optimal level of potassium in broiler diet is 0.72 p. 100 during the first period of age (from 1-30 days) and 0.88 p. 100 afterwards. 


\section{Références bibliographiques}

BAIA Gh., 1972. Alimentatia animalelor domestice, Editura Did. si Ped. Bucuresti, pp. 111-112, 431-434.

C'ristea N., Boisteanu I., Barza Elena, Barbura T., 1978. Fiziologia animalelor domestice, Editura Did. si Ped., Bucuresti, pp. 241-242.

Gürtler H., Ketz N.A., Kolb E., Schroder L., Seidel N., 1975. Physiologie des animaux domestiques, Vigot Frères, Paris, pp. 557-558.

Kolb E., GürtLer H., 1971. Ernährungsphysiologie der landwirtschaftlichen Nutztiere, V.E.B. Gustav Fischer Verlag, Jena, pp. 678-685.

KolB E., 1974. Lehrbuch der Physiologie der Haustiere, V.E.B. Gustav Fischer Verlag, Jena, p. $148,450$.

Martin V., Mihai D., 1979. Dismineralozele Ia animale, Editura «Ceres», Bucuresti, pp. $55-60$. 